\title{
THE CONVERGENCE OF $\alpha$ SCHEMES FOR CONSERVATION LAWS II: FULLY-DISCRETE CASE*
}

\author{
NAN JIANG ${ }^{\dagger}$
}

\begin{abstract}
The entropy convergence of a class of fully-discrete $\alpha$ schemes is shown for scalar convex conservation laws in one dimension. These schemes were constructed by S. Osher and S. Chakravarthy in the mid of 80's [1, 12]. When $m=2$, this class of schemes includes, for different values of $\alpha$, high accuracy (low truncation error) second-order schemes, the conventional second-order accurate upwind total variation diminishing (TVD) scheme and even a third-order accurate TVD scheme. The proof of the entropy consistence is accomplished by using Yang's wavewise entropy inequality (WEI) framework [17]. The convergence of semi-discrete version of $\alpha$ schemes was proven in a companion paper [6].
\end{abstract}

Key words. Conservation laws, fully-discrete $\alpha$ schemes, entropy convergence.

AMS subject classifications. 65M12 (35L60).

1. Introduction. This paper is concerned with entropy convergence of fullydiscrete $\alpha$ schemes, which have been demonstrated to be very successful in solving problems of conservation laws $[1,2,12]$. This class of high-order schemes was formed and analyzed by S. Osher and S. Chakravarthy. For $0<\alpha \leq \frac{1}{2}$ and $m=2,3, \cdots, 8$, the schemes are $2 m-2$ order accurate methods. Along with the construction of the schemes, they have also established the TVD property of this family of schemes. Nonetheless the entropy convergence of these schemes has been open. Previously, the author has shown that, when $m=2, \alpha$ schemes of the semi-discrete version satisfy wavewise entropy inequality (WEI). Consequently, the numerical solutions of $\alpha$ schemes indeed converge to the entropy solution [6]. Our aim, at present, is to show the entropy consistence of fully-discrete $\alpha$ schemes for $m=2$, which include second to third order accurate schemes. The method of this paper is based on Yang's original WEI framework [17] for fully-discrete schemes, of which he has used to establish the convergence of MUSCL schemes for convex problems.

The advantage of this approach is that it does not impose non-homogeneous limitations on the schemes which, on the other hand, is often required by the classical cell entropy inequality (CEI) method. In addition, when applying the WEI convergence criterion of Yang's framework to show the entropy convergence of a particular scheme, one only needs to check the WEI in the rarefaction area. In other words, the shock area has been safely removed from scrutiny in the convergence analysis. Furthermore, even in the rarefaction area, WEI method requires a much weaker condition for the convergence than the traditional CEI approach. Recently, by utilizing the WEI framework, the author [7] is able to establish the extremum traceableness of the general total variation diminishing schemes. Based on this property, the author has shown the entropy convergence of the schemes with van Leer's flux limiter [14], the result is valid for scalar convex conservation laws in one dimensional case. We realize that there are many numerically efficient methods for which the question of convergence is still open. For example, the class of $\beta$ schemes, which are all third-order accurate when $m=2$ in their unlimited version [12]. We will address some of their convergence issues elsewhere.

\footnotetext{
${ }^{*}$ Received January 6, 2013; accepted for publication July 31, 2013.

$\dagger$ Department of Mathematical Sciences, University of South Dakota, Vermillion, SD 57069, USA (njiang@usd.edu).
} 
We consider numerical approximations to the scalar conservation laws

$$
\left\{\begin{array}{l}
u_{t}+f(u)_{x}=0 \\
u(x, 0)=u_{0}(x),
\end{array}\right.
$$

where $f \in C^{1}(\mathbb{R})$ is convex, and $u_{0} \in B V(\mathbb{R})$. Here $B V$ stands for the subspace of $L_{l o c}^{1}$ consisting of functions with bounded total variation. To introduce the numerical methods, we let $\lambda=\frac{\tau}{h}$, where $h$ and $\tau$ are spatial and temporal steps respectively. Denote $u_{k}^{n}=u\left(x_{k}, t_{n}\right)$ be the nodal values of the piecewise constant mesh function $u_{h}(x, t)$ approximating the solution of (1.1). The numerical schemes that we are interested in admit conservative form

$$
u_{k}^{n+1}=H\left(u_{k-p}^{n}, \cdots, u_{k+p}^{n} ; \lambda\right)=u_{k}^{n}-\lambda\left(g_{k+\frac{1}{2}}^{n}-g_{k-\frac{1}{2}}^{n}\right),
$$

where the numerical flux $g$ is given by

$$
g_{k+\frac{1}{2}}^{n}=g_{k+\frac{1}{2}}\left[u_{k}^{n}\right]
$$

and

$$
g_{k+\frac{1}{2}}[v]=g\left(v_{k-p+1}, v_{k-p+2}, \cdots, v_{k}, \cdots, v_{k+p}\right),
$$

for any data $\left\{v_{j}\right\}$.

The function $g$ is Lipschitz continuous with respect to its $2 p$ arguments and is consistent with the conservation law in the sense that

$$
g(u, u, \cdots, u) \equiv f(u)
$$

The paper is organized as follows. In section 2, we review the notions of the extremum paths, the definition of $\alpha$ schemes in the case of $m=2$, and then we establish the extremum traceableness of the general TVD schemes, which is necessary for analyzing the entropy convergence of the schemes that will be given in the next section. In section 3, we present a simplified version of Yang's convergence criterion, an important entropy estimate, and finally the main result.

\section{Extremum traceableness of the TVD schemes.}

2.1. The extremum paths. In this subsection, we introduce the $\alpha$ methods that constructed by Chakravarthy and Osher [1, 12] and the notions of Yang's extremum paths [17].

To improve the readability, throughout the rest of the paper we use the shorthand notations of $f_{k}^{n}:=f\left(u_{k}^{n}\right)$, and $u_{k \pm \frac{1}{2}}^{n}:=\Delta u_{k \pm \frac{1}{2}}^{n}= \pm\left(u_{k \pm 1}^{n}-u_{k}^{n}\right), f_{k \pm \frac{1}{2}}^{n}:=\Delta f_{k \pm \frac{1}{2}}^{n}=$ $\pm\left(f_{k \pm 1}^{n}-f_{k}^{n}\right)$. Also, whenever there is no ambiguity in the context, we employ the simplified notations: $u^{k}:=u_{k}^{n+1}, u_{k}:=u_{k}^{n}, f_{k}:=f_{k}^{n}$, and $f_{k \pm \frac{1}{2}}^{ \pm}:=\left(f_{k \pm \frac{1}{2}}^{n}\right)^{ \pm}$, where $k$ and $n$ are the spatial and temporal indexes respectively. by

Let $g_{k+\frac{1}{2}}^{E}:=g^{E}\left(u_{k}^{n}, u_{k+1}^{n}\right)$ be the flux of an $E$-scheme [13] that is characterized

$$
\operatorname{sgn}\left(u_{k+1}^{n}-u_{k}^{n}\right)\left[g_{k+\frac{1}{2}}^{E}-f(u)\right] \leq 0,
$$


for all $u$ in between $u_{k}^{n}$ and $u_{k+1}^{n}$. Then the flux differences are defined by

$$
f_{k+\frac{1}{2}}^{+}=f_{k+1}-g_{k+\frac{1}{2}}^{E}
$$

and

$$
f_{k+\frac{1}{2}}^{-}=g_{k+\frac{1}{2}}^{E}-f_{k}
$$

At the time level $t=t^{n}$, for all $k$, we define a series of local CFL numbers

$$
\nu_{k+\frac{1}{2}}^{+}=\frac{\lambda f_{k+\frac{1}{2}}^{+}}{u_{k+\frac{1}{2}}}, \quad \nu_{k+\frac{1}{2}}^{-}=\frac{\lambda f_{k+\frac{1}{2}}^{-}}{u_{k+\frac{1}{2}}} .
$$

Clearly, we have $\nu_{k+\frac{1}{2}}^{+} \geq 0$ and $\nu_{k+\frac{1}{2}}^{-} \leq 0$. For convenience, we also set the ratios

$$
r_{k}^{+}=\frac{f_{k-\frac{1}{2}}^{+}}{f_{k+\frac{1}{2}}^{+}}, \quad r_{k}^{-}=\frac{f_{k+\frac{1}{2}}^{-}}{f_{k-\frac{1}{2}}^{-}} .
$$

The "minmod" operator is given by

$$
\operatorname{minmod}(x, y)=\left\{\begin{array}{l}
x, \text { if }|x| \leq|y| \text { and } x y>0 \\
y, \text { if }|x|>|y| \text { and } x y>0 \\
0, \text { if } x y \leq 0
\end{array}\right.
$$

which can be converted to, divided by $x$, a monotone increasing function

$$
\phi(r)=\max (0, \min (1, r))=\left\{\begin{array}{lr}
1, & \text { if } r \geq 1, \\
r, & \text { if } 0 \leq r \leq 1, \\
0, & \text { if } r \leq 0,
\end{array}\right.
$$

with $r=\frac{y}{x}$. Clearly, $\phi(r)$ has a symmetry property

$$
\frac{\phi(r)}{r}=\phi\left(\frac{1}{r}\right)
$$

This property is very helpful to rewriting an $\alpha$ scheme into an increment form. For $0<\alpha \leq \frac{1}{2}$ and $m=2$, an $\alpha$ scheme [1, 12], is given by

$$
u^{k}=u_{k}-\lambda\left(g_{k+\frac{1}{2}}-g_{k-\frac{1}{2}}\right)
$$

where

$$
\begin{aligned}
g_{k+\frac{1}{2}} & =g_{k+\frac{1}{2}}^{E}-\alpha\left(f_{k+\frac{3}{2}}^{-}\right)^{(1)}-\left(\frac{1}{2}-\alpha\right)\left(f_{k+\frac{1}{2}}^{-}\right)^{(0)} \\
& +\left(\frac{1}{2}-\alpha\right)\left(f_{k+\frac{1}{2}}^{+}\right)^{(0)}+\alpha\left(f_{k-\frac{1}{2}}^{+}\right)^{(-1)} .
\end{aligned}
$$

The superscripts shown over the $f^{ \pm}$denote flux limited values of $f^{ \pm}$, and are computed as follows: 


$$
\begin{aligned}
\left(f_{k+\frac{3}{2}}^{-}\right)^{(1)} & =\operatorname{minmod}\left[f_{k+\frac{3}{2}}^{-}, b f_{k+\frac{1}{2}}^{-}\right] \\
& =\operatorname{minmod}\left[\frac{1}{b} \frac{f_{k+\frac{3}{2}}^{-}}{f_{k+\frac{1}{2}}^{-}}, 1\right] b f_{k+\frac{1}{2}}^{-} \\
& =\phi\left(\frac{r_{k+1}^{-}}{b}\right) b f_{k+\frac{1}{2}}^{-}
\end{aligned}
$$

$$
\begin{aligned}
\left(f_{k+\frac{1}{2}}^{-}\right)^{(0)} & =\operatorname{minmod}\left[f_{k+\frac{1}{2}}^{-}, b f_{k+\frac{3}{2}}^{-}\right] \\
& =\operatorname{minmod}\left[1, b \frac{f_{k+\frac{3}{2}}^{-}}{f_{k+\frac{1}{2}}^{-}}\right] f_{k+\frac{1}{2}}^{-} \\
& =\phi\left(b r_{k+1}^{-}\right) f_{k+\frac{1}{2}}^{-}
\end{aligned}
$$

$$
\begin{aligned}
\left(f_{k+\frac{1}{2}}^{+}\right)^{(0)} & =\operatorname{minmod}\left[f_{k+\frac{1}{2}}^{+}, b f_{k-\frac{1}{2}}^{+}\right] \\
& =\operatorname{minmod}\left[1, b \frac{f_{k-\frac{1}{2}}^{+}}{f_{k+\frac{1}{2}}^{+}}\right] f_{k+\frac{1}{2}}^{+} \\
& =\phi\left(b r_{k}^{+}\right) f_{k+\frac{1}{2}}^{+} \\
\left(f_{k-\frac{1}{2}}^{+}\right)^{(-1)} & =\operatorname{minmod}\left[f_{k-\frac{1}{2}}^{+}, b f_{k+\frac{1}{2}}^{+}\right] \\
& =\operatorname{minmod}\left[\frac{1}{b} \frac{f_{k-\frac{1}{2}}^{+}}{f_{k+\frac{1}{2}}^{+}}, 1\right] b f_{k+\frac{1}{2}}^{+} \\
& =\phi\left(\frac{r_{k}^{+}}{b}\right) b f_{k+\frac{1}{2}}^{+}
\end{aligned}
$$

In the above, $b$ is a "compression" parameter chosen in the range

$$
0<b \leq 1+\frac{1}{2 \alpha}=b_{\max }
$$

We shall assume for the remainder of the paper that the local CFL numbers satisfy $\left|\nu_{k+\frac{1}{2}}^{ \pm}\right| \leq 1$ for all $k \in \mathbb{Z}$.

By rewriting the expression

$$
\left[\left(\frac{1}{2}-\alpha\right) \phi\left(b r_{k}^{+}\right)+\alpha b \phi\left(\frac{r_{k}^{+}}{b}\right)\right] \nu_{k+\frac{1}{2}}^{+} u_{k+\frac{1}{2}}
$$

as

$$
\left[\left(\frac{1}{2}-\alpha\right) \frac{1}{r_{k}^{+}} \phi\left(b r_{k}^{+}\right)+\alpha b \frac{1}{r_{k}^{+}} \phi\left(\frac{r_{k}^{+}}{b}\right)\right] \nu_{k-\frac{1}{2}}^{+} u_{k-\frac{1}{2}} ;
$$


and

$$
\left[\alpha b \phi\left(\frac{r_{k}^{-}}{b}\right)+\left(\frac{1}{2}-\alpha\right) \phi\left(b r_{k}^{-}\right)\right] \nu_{k-\frac{1}{2}}^{-} u_{k-\frac{1}{2}}
$$

as

$$
\left[\alpha b \frac{1}{r_{k}^{-}} \phi\left(\frac{r_{k}^{-}}{b}\right)+\left(\frac{1}{2}-\alpha\right) \frac{1}{r_{k}^{-}} \phi\left(b r_{k}^{-}\right)\right] \nu_{k+\frac{1}{2}}^{-} u_{k+\frac{1}{2}},
$$

it is easy to see that the schemes (2.9)-(2.10) can be written in an increment form

$$
u^{k}=u_{k}-C_{k-\frac{1}{2}} u_{k-\frac{1}{2}}+D_{k+\frac{1}{2}} u_{k+\frac{1}{2}}
$$

with

$$
\begin{aligned}
C_{k-\frac{1}{2}} & =\nu_{k-\frac{1}{2}}^{+}\left[\left(\frac{1}{2}-\alpha\right) \frac{1}{r_{k}^{+}} \phi\left(b r_{k}^{+}\right)+\alpha b \frac{1}{r_{k}^{+}} \phi\left(\frac{r_{k}^{+}}{b}\right)\right. \\
& \left.+1-\left(\frac{1}{2}-\alpha\right) \phi\left(b r_{k-1}^{+}\right)-\alpha b \phi\left(\frac{r_{k-1}^{+}}{b}\right)\right]
\end{aligned}
$$

and

$$
\begin{aligned}
D_{k+\frac{1}{2}} & =-\nu_{k+\frac{1}{2}}^{-}\left[1-\alpha b \phi\left(\frac{r_{k+1}^{-}}{b}\right)-\left(\frac{1}{2}-\alpha\right) \phi\left(b r_{k+1}^{-}\right)\right. \\
& \left.+\alpha b \frac{1}{r_{k}^{-}} \phi\left(\frac{r_{k}^{-}}{b}\right)+\left(\frac{1}{2}-\alpha\right) \frac{1}{r_{k}^{-}} \phi\left(b r_{k}^{-}\right)\right] .
\end{aligned}
$$

This form of the schemes provides a convenient way of checking extremum traceability and TVD property of the schemes [7, 5].

The concept of discrete extremum paths was introduced by Yang (see Definition $6.3[16]$ and Definition 2.13 [17]) for both semi-discrete and fully-discrete cases. For the convenience of applications, we restate the relevant definitions of the fully-discrete case in order.

Consider a numerical solution $u$ defined on the set of grid points $X:=\left\{\left(x_{j}, t_{n}\right)\right.$ : $\left.j \in \mathbb{Z}, n \in \mathbb{Z}^{+}\right\}$. A finite set of successive grid points $\left\{x_{q}, \cdots, x_{r}\right\}$ with $r \geq q$ is said to be the stencil of a spatial maximum, or simply an $\bar{E}$-stencil of $u$ at the time $t_{n}$, provided $u_{q}^{n}=\cdots=u_{r}^{n}, u_{q-1}^{n}<u_{q}^{n}$ and $u_{r+1}^{n}<u_{r}^{n}$. Notions of $\underline{E}$-stencils for minima and E-stencils for general extrema are defined similarly. Throughout the paper, we refer to [17] for the definitions, lemmas and theorems that we have quoted in the context.

Definition 2.1 (see Definition 2.13 [17]). A nonempty subset of $X$ denoted by $\bar{E}_{t_{n}, t_{m}}, n \leq m$, is called a ridge of the numerical solution $u$ from $t_{n}$ to $t_{m}$ if

(i) for all $\nu, n \leq \nu \leq m$, the set

$$
P_{\bar{E}}(\nu):=\left\{x_{j}:\left(x_{j}, t_{\nu}\right) \in \bar{E}_{t_{n}, t_{m}}\right\}=\left\{x_{q^{\nu}}, \cdots, x_{r^{\nu}}\right\}
$$

is not empty and is an $\bar{E}$-stencil of $u$ at $t_{\nu}$;

(ii) for all $\nu, n \leq \nu \leq m-1$,

$$
P_{\bar{E}}(\nu) \cup P_{\bar{E}}(\nu+1)=\left\{x_{j}: \min \left(q^{\nu}, q^{\nu+1}\right) \leq j \leq \max \left(r^{\nu}, r^{\nu+1}\right)\right\} .
$$


The set $P_{\bar{E}}(\nu)$ is called the $x$-projection of $\bar{E}_{t_{n}, t_{m}}$ at $t_{\nu}$. The value of $u$ along the ridge is denoted by $V_{\bar{E}}(\nu): V_{\bar{E}}(\nu)=u_{j}^{\nu}$ for $q^{\nu} \leq j \leq r^{\nu}$.

If, for all $\nu, n \leq \nu \leq m$, the $\bar{E}$-stencil in the item (i) of the definition is replaced by an $\underline{E}$-stencil, then the set is called a trough of $u$ from $t_{n}$ to $t_{m}$ and is denoted by $\underline{E}_{t_{n}, t_{m}}$. The related notions $P_{\underline{E}}(\nu)$ and $V_{\underline{E}}(\nu)$ are defined similarly. Ridges and troughs are also called extremum paths. When we do not distinguish between ridges and troughs, we use $E_{t_{n}, t_{m}}, P_{E}(\nu)$, and $V_{E}(\nu)$ for either type. We write

$$
E_{t_{n}, t_{m}}^{1}<(\leq) E_{t_{n}, t_{m}}^{2} \text {, if } \max P_{E^{1}}(\nu)<(\leq) \max P_{E^{2}}(\nu) \text { for } n \leq \nu \leq m \text {. }
$$

Definition 2.2 (see Definition 2.14 [17]). A scheme is said to be extremum traceable if there exists a positive constant $c \geq 1$ such that for each numerical solution $u$ of the scheme and each integer $N>0$, there exists a finite or infinite collection of extremum paths $\left\{E_{t_{0}, t_{N}}^{l}\right\}_{l=l_{1}}^{l_{2}}$ with the following properties:

(i) $\left\{P_{E^{l}}(N)\right\}_{l=l_{1}}^{l_{2}}$ is precisely the set of $E$-stencils of $u_{j}^{n}$ at the time $t_{N}$ arranged in ascending spatial coordinates.

(ii) If $E_{t_{0}, t_{N}}^{l}$ is a ridge (trough), then $V_{E_{l}}(n)$ is a non increasing (non decreasing) function of $n$. then

(iii) Let $P_{E^{l}}(n)=\left\{x_{q^{l}(n)}, \cdots, x_{r^{l}(n)}\right\}$ for $1 \leq n \leq N$. If $P_{E^{l}}(n) \cap P_{E^{l}}(n+1)=\emptyset$,

$$
\begin{aligned}
& \left|u_{q^{l}(n+1)}^{n}-u_{r^{l}(n)}^{n}\right| \leq c\left|V_{E^{l}}(n+1)-V_{E^{l}}(n)\right| \quad \text { when } \quad q^{l}(n+1)>r^{l}(n), \\
& \left|u_{r^{l}(n+1)}^{n}-u_{q^{l}(n)}^{n}\right| \leq c\left|V_{E^{l}}(n+1)-V_{E^{l}}(n)\right| \quad \text { when } \quad q^{l}(n)>r^{l}(n+1) .
\end{aligned}
$$

(iv) If $l_{2}>l_{1}$, then $E_{t_{0}, t_{N}}^{l}<E_{t_{0}, t_{N}}^{l+1}$ for $l_{1} \leq l \leq l_{2}-1$.

2.2. Extremum traceableness of the TVD schemes. Since the true solution of (1.1) has the TVD property, it is very desirable to maintain the TVD property in the design of a numerical scheme that approximating scalar conservation laws (1.1). The schemes equipped with the TVD property will prevent new extrema values (that generate spurious oscillations of the solutions) other than those which propagate from the previous time-level. Besides that, we have confidence in the numerical solutions computed by such scheme, for it will at least converge to a weak solution $[10,11]$ of (1.1). In the next section, we will use Yang's WEI convergence criterion to show the entropy convergence of the schemes of (2.9)-(2.10). For that purpose, we need to verify the schemes are extremum traceable. By focusing on the general TVD schemes, we are able to show the following result.

ThEOREM 2.3 (see Theorem 2.3 [7]). The sufficient conditions for the schemes (2.9)-(2.10) to be extremum traceable are the following inequalities:

$$
0 \leq C_{k+\frac{1}{2}}, \quad 0 \leq D_{k+\frac{1}{2}}, \quad 0 \leq C_{k+\frac{1}{2}}+D_{k+\frac{1}{2}} \leq 1, \text { for all } k
$$

there is a positive constant $\mu$ such that, if $u_{k}$ is a space extremum, then

$$
\max \left\{C_{k \pm \frac{1}{2}}, C_{k \pm \frac{3}{2}}, D_{k \pm \frac{1}{2}}, D_{k+\frac{3}{2}}\right\} \leq \frac{\mu}{4}<\frac{1}{4},
$$

where $C_{k+\frac{1}{2}}$ and $D_{k+\frac{1}{2}}$ are given by (2.16)-(2.17). 
The inequalities of (2.18) are the well know sufficient conditions for the schemes (2.9)-(2.10) to be TVD, introduced by Harten [5] in 1983. In the following corollary, we reformulate the conclusions of the Theorem 2.3 in the language of the local CFL numbers.

Corollary 2.4. The sufficient conditions for the schemes (2.9)-(2.10) to be extremum traceable are the following inequalities:

$$
\nu_{k+\frac{1}{2}}^{+}-\nu_{k+\frac{1}{2}}^{-} \leq \frac{4 \alpha}{1+4 \alpha}
$$

for all $k$ and for $0<\alpha \leq \frac{1}{2}$; when $u_{k}$ is an extremum,

$$
\max \left(\nu_{k-\frac{1}{2}}^{+},-\nu_{k+\frac{1}{2}}^{-}\right)<\frac{\alpha}{2},
$$

and

$$
\max \left\{\nu_{k+\frac{1}{2}}^{+}, \nu_{k \pm \frac{3}{2}}^{+},-\nu_{k+\frac{3}{2}}^{-},-\nu_{k-\frac{1}{2}}^{-}\right\} \leq \frac{\alpha}{2} \frac{4 \alpha}{1+4 \alpha}
$$

Proof. Indeed, for all $k$, in reference of (2.16)-(2.17), we have $C_{k+\frac{1}{2}} \geq 0, D_{k+\frac{1}{2}} \geq$ 0 , and

$$
C_{k+\frac{1}{2}}+D_{k+\frac{1}{2}} \leq\left(\nu_{k+\frac{1}{2}}^{+}-\nu_{k+\frac{1}{2}}^{-}\right) \frac{4 \alpha+1}{4 \alpha} \leq 1
$$

When $u_{k}$ is an extremum, $C_{k \pm \frac{1}{2}}$, and $D_{k \pm \frac{1}{2}}$ are reduced to

$$
\begin{gathered}
C_{k-\frac{1}{2}}=\nu_{k-\frac{1}{2}}^{+}\left[1-\left(\frac{1}{2}-\alpha\right) \phi\left(b r_{k-1}^{+}\right)-\alpha b \phi\left(\frac{r_{k-1}^{+}}{b}\right)\right] \leq \nu_{k-\frac{1}{2}}^{+}, \\
D_{k+\frac{1}{2}}=-\nu_{k+\frac{1}{2}}^{-}\left[1-\alpha b \phi\left(\frac{r_{k+1}^{-}}{b}\right)-\left(\frac{1}{2}-\alpha\right) \phi\left(b r_{k+1}^{-}\right)\right] \leq-\nu_{k+\frac{1}{2}}^{-}, \\
C_{k+\frac{1}{2}}=\nu_{k+\frac{1}{2}}^{+}\left[1+\left(\frac{1}{2}-\alpha\right) \frac{1}{r_{k+1}^{+}} \phi\left(b r_{k+1}^{+}\right)+\alpha b \frac{1}{r_{k+1}^{+}} \phi\left(\frac{r_{k+1}^{+}}{b}\right)\right] \leq \nu_{k+\frac{1}{2}}^{+} \frac{4 \alpha+1}{4 \alpha}
\end{gathered}
$$

and

$$
D_{k-\frac{1}{2}}=-\nu_{k-\frac{1}{2}}^{-}\left[1+\alpha b \frac{1}{r_{k-1}^{-}} \phi\left(\frac{r_{k-1}^{-}}{b}\right)+\left(\frac{1}{2}-\alpha\right) \frac{1}{r_{k-1}^{-}} \phi\left(b r_{k-1}^{-}\right)\right] \leq-\nu_{k-\frac{1}{2}}^{-} \frac{4 \alpha+1}{4 \alpha} .
$$
tions

Following the four inequalities of the above, we have arrived the desired estima-

$$
\begin{gathered}
C_{k \pm \frac{1}{2}}+D_{k \pm \frac{1}{2}} \leq\left(\nu_{k \pm \frac{1}{2}}^{+}-\nu_{k \pm \frac{1}{2}}^{-}\right) \frac{4 \alpha+1}{4 \alpha} \leq 1 \\
2 C_{k-\frac{1}{2}}+D_{k-\frac{1}{2}} \leq 2 \nu_{k-\frac{1}{2}}^{+}-\nu_{k-\frac{1}{2}}^{-} \frac{4 \alpha+1}{4 \alpha}<1,
\end{gathered}
$$




$$
\begin{gathered}
C_{k+\frac{1}{2}}+2 D_{k+\frac{1}{2}} \leq \nu_{k+\frac{1}{2}}^{+} \frac{4 \alpha+1}{4 \alpha}-2 \nu_{k+\frac{1}{2}}^{-}<1, \\
C_{k+\frac{1}{2}}+D_{k+\frac{1}{2}}+D_{k+\frac{3}{2}}+C_{k-\frac{1}{2}} \\
\leq\left(\nu_{k+\frac{1}{2}}^{+}-\nu_{k+\frac{3}{2}}^{-}\right) \frac{4 \alpha+1}{4 \alpha}+\nu_{k-\frac{1}{2}}^{+}-\nu_{k+\frac{1}{2}}^{-}<1,
\end{gathered}
$$

and

$$
\begin{aligned}
& C_{k-\frac{1}{2}}+D_{k-\frac{1}{2}}+D_{k+\frac{1}{2}}+C_{k-\frac{3}{2}} \\
\leq & \nu_{k-\frac{1}{2}}^{+}-\nu_{k-\frac{1}{2}}^{-} \frac{4 \alpha+1}{4 \alpha}-\nu_{k+\frac{1}{2}}^{-}+\nu_{k-\frac{3}{2}}^{+} \frac{4 \alpha+1}{4 \alpha}<1,
\end{aligned}
$$

and so on.

Notice that $\max _{0<\alpha \leq \frac{1}{2}} \frac{\alpha}{2} \frac{4 \alpha}{1+4 \alpha}=\frac{1}{6}$, we have the following lemma when the building block of the $\alpha$ schemes belongs to a subfamily of $E$-fluxes that defined by

$$
g^{E}(x, y)= \begin{cases}f(x) & \text { if } s \leq x \leq y \\ f(y) & \text { if } x \leq y \leq s\end{cases}
$$

where $s$ is a sonic point of $f(\cdot): f^{\prime}(s)=0$.

It is clear that both Godunov [4] and Engquist-Osher [3] fluxes:

$$
g^{G o d}\left(u_{j}, u_{j+1}\right)=\left\{\begin{array}{lll}
\min _{u_{j} \leq w \leq u_{j+1}} f(w) & \text { when } & u_{j} \leq u_{j+1} \\
\max _{u_{j} \geq w \geq u_{j+1}} f(w) & \text { when } & u_{j} \geq u_{j+1}
\end{array}\right.
$$

and

$$
g^{E O}\left(u_{j}, u_{j+1}\right)=\int_{0}^{u_{j}} \max \left(f^{\prime}(w), 0\right) d w+\int_{0}^{u_{j+1}} \min \left(f^{\prime}(w), 0\right) d w+f(0)
$$

are members of the fluxes given by (2.23).

Lemma 2.5. The schemes (2.9)-(2.10) with the building block of a E-flux defined by $(2.23)$ are extremum traceable, provided that

$$
\nu_{k+\frac{1}{2}}^{+}-\nu_{k+\frac{1}{2}}^{-} \leq \frac{4 \alpha}{1+4 \alpha}
$$

for all $k$ and for $0<\alpha \leq \frac{1}{2}$; when $u_{k}$ is an extremum, we have $\lambda K^{\prime}=$ $\lambda \max _{u_{k-2} \leq w \leq u_{k+2}}\left|f^{\prime}(w)\right| \leq \frac{1}{6}$.

3. The convergence of $\alpha$ schemes. The schemes that are concerned will satisfy the following separation property at the spatial extrema. It characterizes that, in the next time level, the values of maximum (minimum) values of the numerical solutions are not increasing (decreasing). E. Tadmor [15] presented the similar kind of conditions that have been used to check TVD conditions of a scheme conveniently. 
Assumption 3.1. The numerical fluxes $g_{k+\frac{1}{2}}^{n},-\infty<k<\infty$, satisfy

$$
g_{k+\frac{1}{2}}^{n} \geq f\left(u_{k}^{n}\right) \geq g_{k-\frac{1}{2}}^{n} \quad \text { if } \quad u_{k}^{n} \geq u_{k \pm 1}^{n}
$$

and

$$
g_{k+\frac{1}{2}}^{n} \leq f\left(u_{k}^{n}\right) \leq g_{k-\frac{1}{2}}^{n} \quad \text { if } \quad u_{k}^{n} \leq u_{k \pm 1}^{n}
$$

Lemma 3.2. The scheme (2.9)-(2.10) satisfies the Assumption 3.1.

Proof. If $u_{k} \geq u_{k \pm 1}$, then

$$
\begin{aligned}
g_{k+\frac{1}{2}} & =g_{k+\frac{1}{2}}^{E}-\alpha\left(f_{k+\frac{3}{2}}^{-}\right)^{(1)}-\left(\frac{1}{2}-\alpha\right)\left(f_{k+\frac{1}{2}}^{-}\right)^{(0)} \\
& \geq g_{k+\frac{1}{2}}^{E}-\alpha b f_{k+\frac{1}{2}}^{-}-\left(\frac{1}{2}-\alpha\right) f_{k+\frac{1}{2}}^{-} \\
& \geq g_{k+\frac{1}{2}}^{E}+\left[-\left(\alpha+\frac{1}{2}\right)-\left(\frac{1}{2}-\alpha\right)\right] f_{k+\frac{1}{2}}^{-} \\
& =f_{k}
\end{aligned}
$$

and

$$
\begin{aligned}
g_{k-\frac{1}{2}} & =g_{k-\frac{1}{2}}^{E}+\left(\frac{1}{2}-\alpha\right)\left(f_{k-\frac{1}{2}}^{+}\right)^{(0)}+\alpha\left(f_{k-\frac{3}{2}}^{+}\right)^{(-1)} \\
& \leq g_{k-\frac{1}{2}}^{E}+\left[\left(\frac{1}{2}-\alpha\right)+\alpha b\right] f_{k-\frac{1}{2}}^{+} \\
& \leq g_{k-\frac{1}{2}}^{E}+f_{k-\frac{1}{2}}^{+} \\
& =f_{k} .
\end{aligned}
$$

Similarly, we can show that if $u_{k}^{n} \leq u_{k \pm 1}^{n}$, then $g_{k+\frac{1}{2}}^{n} \leq f\left(u_{k}^{n}\right) \leq g_{k-\frac{1}{2}}^{n}$.

In reference of $(1.2)$, we denote $\tilde{v}_{j}=H\left(v_{j-p}, \cdots, v_{j+p} ; \lambda\right)$ and $\bar{v}_{j}=\frac{v_{j}+\tilde{v}_{j}}{2}$ for any collection of data $\left\{v_{j}\right\}$. Let $\bar{v}_{j \pm \frac{1}{2}}^{n}:=\Delta \bar{v}_{j \pm \frac{1}{2}}^{n}, \tilde{v}_{j \pm \frac{1}{2}}^{n}:=\Delta \tilde{v}_{j \pm \frac{1}{2}}^{n}$, and $f[w ; L, R]$ be the linear function interpolating $f(w)$ at $w=L$ and $w=R$. In this section, we assume that $f^{\prime \prime}(w) \geq 0$.

Definition 3.3 (see Definition $2.20[17]$ ). We call an ordered pair of numbers $\{L, R\}$ a rarefying pair if $L<R$ and $f[w ; L, R]>f(w)$ when $L<w<R$. We call a collection of data $\Gamma=\left\{v_{j}\right\}_{j=I-p}^{J+p}$ an $\varepsilon$-rarefying collection of the scheme to the rarefying pair $\{L, R\}$ if, for $\varepsilon>0$,

(i) $L=v_{I} \leq v_{I+1} \leq \cdots \leq v_{J}=R$;

(ii) $\tilde{v}_{I} \leq \tilde{v}_{I+1} \leq \cdots \leq \tilde{v}_{J},\left|L-\tilde{v}_{I}\right|<\varepsilon,\left|R-\tilde{v}_{J}\right|<\varepsilon$;

(iii) either $v_{I-1} \geq v_{I}$ or $v_{I}=v_{I+1}$; and either $v_{J+1} \leq v_{J}$ or $v_{J-1}=v_{J}$.

Clearly, the conditions of (i) and (ii) imply that

$$
\bar{v}_{I} \leq \bar{v}_{I+1} \leq \cdots \leq \bar{v}_{J},\left|L-\bar{v}_{I}\right|<\frac{\varepsilon}{2}, \text { and }\left|R-\bar{v}_{J}\right|<\frac{\varepsilon}{2} .
$$

We define the piecewise constant function $g_{\Gamma}$ associated with the $\varepsilon$-rarefying collection $\Gamma$ as follows:

$$
g_{\Gamma}(w)=g_{j+\frac{1}{2}}[v] \quad \text { for } w \in\left(\bar{v}_{j}, \bar{v}_{j+1}\right), \quad I \leq j \leq J-1 .
$$


A 0-rarefying collection $\Gamma=\left\{v_{j}\right\}_{j=I-2}^{J+2}$ of the scheme to the pair $\{L, R\}$ that satisfies

$$
L=v_{I-2}=v_{I-1}=v_{I}=v_{I+1} \leq \cdots \leq v_{J-1}=v_{J}=v_{J+1}=v_{J+2}=R
$$

is called a normal collection.

TheOREm 3.4 (see Theorem 2.21[17]). An extremum traceable scheme that satisfies Assumption 3.1 converges for convex conservation laws if, for every rarefying pair $\{L, R\}$ and $\varepsilon$-rarefying collection to the pair,

$$
\int_{L}^{R} f[w ; L, R] d w-\int_{\bar{v}_{I}}^{\bar{v}_{J}} g_{\Gamma}(w) d w>\delta
$$

for some constant $\delta>0$ depending only on the exact flux $f$, the numerical flux function $g$, and the two numbers $L$ and $R$, provided that $\varepsilon$ is sufficiently small.

For the class of $\alpha$ schemes concerned, this convergence criterion can be simplified by the following Lemma.

Lemma 3.5. An extremum traceable scheme of the form (2.9)-(2.10) converges for convex conservation laws, provided that for each rarefying pair $\{L, R\}$ there is a constant $\delta>0$ such that the inequality (3.3) holds for all normal collections of the scheme to the pair $\{L, R\}$.

Proof. Let $\Lambda=\left\{\kappa_{P-2}, \cdots, \kappa_{Q+2}\right\}$ be an arbitrary $\varepsilon$-rarefying collection of the scheme to the pair $\{L, R\}$. Let

$$
S^{\prime}=\int_{\bar{\kappa}_{P}}^{\bar{\kappa}_{Q}} g_{\Lambda}(w) d w=\sum_{j=P}^{Q-1}\left(\bar{\kappa}_{j+1}-\bar{\kappa}_{j}\right) g_{j+\frac{1}{2}}[\kappa] .
$$

by (i) and (iii) of Definition 3.3, either $\kappa_{P}$ or $\kappa_{P+1}$ is a minimum. In either case, Assumption 3.1 and the condition (ii) of Definition 3.3 imply that

$$
\varepsilon>\left|L-\tilde{\kappa}_{P}\right|=\left|\tilde{\kappa}_{P}-\kappa_{P}\right|=\lambda\left|g_{P+\frac{1}{2}}[\kappa]-g_{P-\frac{1}{2}}[\kappa]\right| \geq \lambda\left|g_{P \pm \frac{1}{2}}[\kappa]-f(L)\right| .
$$

Similarly, we have

$$
\varepsilon>\left|R-\tilde{\kappa}_{Q}\right| \geq \lambda\left|g_{Q \pm \frac{1}{2}}[\kappa]-f(R)\right| .
$$

Next, we construct a normal collection $\Gamma=\left\{v_{j}\right\}_{j=I-2}^{J+2}$ as follows. First, let $I=P-1$ and $J=Q+1$ and we also set $v_{I-2}=v_{I-1}=v_{I}=L, v_{J}=v_{J+1}=v_{J+2}=R$, and $v_{j}=\kappa_{j}$ for $I+1 \leq j \leq J-1$. Then, we have

$$
g_{I \pm \frac{1}{2}}[v]=f(L) \quad \text { and } \quad g_{J \pm \frac{1}{2}}[v]=f(R),
$$

which imply that,

$$
\bar{v}_{I}=\tilde{v}_{I}=v_{I}=L, \quad \bar{v}_{J}=\tilde{v}_{J}=v_{J}=R .
$$

Thus, the normality of $\Gamma=\left\{v_{j}\right\}_{j=I-2}^{J+2}$ is justified by the non-decreasing relation of

$$
\tilde{v}_{I} \leq \tilde{v}_{I+1} \leq \cdots \leq \tilde{v}_{J}
$$


Indeed, we notice that the following relationship

$$
\tilde{v}_{I+3} \leq \tilde{v}_{I+4} \leq \cdots \leq \tilde{v}_{J-4} \leq \tilde{v}_{J-3}
$$

is directly inherited from the condition (ii) of the given $\varepsilon$-rarefying collection of $\Lambda$

$$
\tilde{\kappa}_{P+2} \leq \tilde{\kappa}_{P+3} \leq \cdots \leq \tilde{\kappa}_{Q-3} \leq \tilde{\kappa}_{Q-2} .
$$

Therefore, we only need to verify that

$$
\tilde{v}_{I} \leq \tilde{v}_{I+1} \leq \tilde{v}_{I+2} \leq \tilde{v}_{I+3}
$$

and

$$
\tilde{v}_{J-3} \leq \tilde{v}_{J-2} \leq \tilde{v}_{J-1} \leq \tilde{v}_{J}
$$

The proof is straight forward and we omit the details.

Secondly, let $G$ be the Lipschitz constant of the numerical flux $g$, and $K=$ $\max \{|f(L)|,|f(R)|\}+G(R-L)$. Denote

$$
S=\int_{L}^{R} g_{\Gamma}(w) d w=\sum_{j=I}^{J-1}\left(\bar{v}_{j+1}-\bar{v}_{j}\right) g_{j+\frac{1}{2}}[v],
$$

then a-priori estimate $\left|S-S^{\prime}\right| \leq 3 K \varepsilon$ holds. Let $\delta^{\prime}$ be a constant such that for all normal collections of the scheme to the pair $\{L, R\}$ the inequality (3.3) holds for $\delta=\delta^{\prime}$. Thus, for $\delta=\delta^{\prime}$, the inequality (3.3) also holds for the normal collection $\Gamma=\left\{v_{j}\right\}_{j=I-2}^{J+2}$. Therefore, for $\delta=\frac{\delta^{\prime}}{2}$, the inequality (3.3) holds for all $\varepsilon$-collection of the scheme to the pair $\{L, R\}$ provided that $\varepsilon \leq \frac{\delta}{3 K}$.

It remains to show the a-priori estimate. First, we notice that $\bar{\kappa}_{j}=\bar{v}_{j}$ for $P+2 \leq$ $j \leq Q-3$, and we can verify that for $P+1 \leq j \leq Q-2$ the $j$ th term of $S$ equals to the $j$ th term of $S^{\prime}$. Therefore the terms of the difference

$$
S-S^{\prime}=\sum_{j=I}^{J-1}\left(\bar{v}_{j+1}-\bar{v}_{j}\right) g_{j+\frac{1}{2}}[v]-\sum_{j=P}^{Q-1}\left(\bar{\kappa}_{j+1}-\bar{\kappa}_{j}\right) g_{j+\frac{1}{2}}[\kappa]
$$

from $j=P+1$ to $j=Q-2$ are all diminished. Then, for the remaining terms, we use the relationship of $\Lambda$ and $\Gamma$ and (3.5)-(3.8) to yield the following estimates.

$$
\begin{gathered}
\left|\bar{v}_{I+1}-\bar{\kappa}_{I+1}\right|<\frac{\varepsilon}{2}, \quad\left|\bar{v}_{J-1}-\bar{\kappa}_{J-1}\right|<\frac{\varepsilon}{2} \\
\left|\bar{v}_{I+1}-\bar{v}_{I}\right|=\left|\bar{v}_{I+1}-L\right| \leq\left|\bar{v}_{I+1}-\bar{\kappa}_{I+1}\right|+\left|\bar{\kappa}_{I+1}-L\right|<\varepsilon,
\end{gathered}
$$

and

$$
\left|\bar{v}_{J}-\bar{v}_{J-1}\right|=\left|\bar{v}_{J-1}-R\right| \leq\left|\bar{v}_{J-1}-\bar{\kappa}_{J-1}\right|+\left|\bar{\kappa}_{Q}-R\right|<\varepsilon .
$$

Finally, using the fact that $\bar{v}_{I+2}=\bar{\kappa}_{P+1}, \bar{v}_{J-2}=\bar{\kappa}_{Q-1}, g_{I+\frac{3}{2}}[v]=g_{P+\frac{1}{2}}[\kappa], g_{J-\frac{3}{2}}[v]=$ $g_{Q-\frac{1}{2}}[\kappa]$, and (3.10)-(3.12), we have derived the desired estimate as follows.

$$
\begin{aligned}
\left|S-S^{\prime}\right| & =\mid\left(\bar{v}_{I+1}-\bar{v}_{I}\right) g_{I+\frac{1}{2}}[v]+\left(\bar{v}_{J}-\bar{v}_{J-1}\right) g_{J-\frac{1}{2}}[v] \\
& +\left(\bar{v}_{I+2}-\bar{v}_{I+1}\right) g_{I+\frac{3}{2}}[v]-\left(\bar{\kappa}_{P+1}-\bar{\kappa}_{P}\right) g_{P+\frac{1}{2}}[\kappa] \\
& +\left(\bar{v}_{J-1}-\bar{v}_{J-2}\right) g_{J-\frac{3}{2}}[v]-\left(\bar{\kappa}_{Q}-\bar{\kappa}_{Q-1}\right) g_{Q-\frac{1}{2}}[\kappa] \mid \\
& \leq\left|\bar{v}_{I+1}-\bar{v}_{I}\right|\left|g_{I+\frac{1}{2}}[v]\right|+\left|\bar{v}_{J}-\bar{v}_{J-1}\right|\left|g_{J-\frac{1}{2}}[v]\right| \\
& +\left|\bar{v}_{I+1}-\bar{\kappa}_{P}\right|\left|g_{I+\frac{3}{2}}[v]\right|+\left|\bar{v}_{J-1}-\bar{\kappa}_{Q}\right|\left|g_{Q-\frac{1}{2}}[\kappa]\right| \\
& <\left(\varepsilon+\varepsilon+\frac{1}{2} \varepsilon+\frac{1}{2} \varepsilon\right) K=3 K \varepsilon
\end{aligned}
$$


and the proof is completed.

For a normal collection $\Gamma=\left\{v_{j}\right\}_{j=I-2}^{J+2}$, we denote the vertex $\left(v_{j}, f\left(v_{j}\right)\right)$ by $V_{j}$ and the area of convex polygon $V_{j_{1}} V_{j_{2}} \cdots V_{j_{r}}$ by $S_{j_{1}, \ldots, j_{r}}$. Let $\sigma_{\Gamma}=\max _{I-2 \leq j \leq J+2}\left|\nu_{j \pm \frac{1}{2}}^{ \pm}\right|$, and let

$$
\alpha_{j}= \begin{cases}0.5 & \text { if } \Delta v_{j-2}=\Delta v_{j+1}=0 \\ 1 & \text { otherwise }\end{cases}
$$

When the building block of the schemes (2.9)-(2.10) is the subclass of $E$-schemes with the fluxes defined by $(2.23)$, we have the following very important inequality, which will enable us to prove Theorem 3.8, the main result of the paper.

Lemma 3.6. Let $\Gamma=\left\{v_{j}\right\}_{j=I-2}^{J+2}$ be a normal collection to a rarefying pair $\{L, R\}$. Then the numerical solutions of the schemes (2.9)-(2.10) for convex conservation laws (1.1) satisfy, for a sufficiently small $\sigma_{\Gamma}$, the following inequality

$$
\int_{L}^{R}\left(f[w ; L, R]-g_{\Gamma}\right) d w \geq S_{I, I+1, \ldots, J}-\sum_{j=I+1}^{J-1} \alpha_{j} S_{j-1, j, j+1} .
$$

Lemma 3.7 ( see Lemma $3.7[17]$ ). We have

$$
S_{I, I+1, \ldots, J}-\sum_{j=I+1}^{J-1} S_{j-1, j, j+1} \geq S_{I, i, i+1, J}-\left(S_{I, i, i+1}+S_{i, i+1, J}\right)
$$

for $I<i<J-1$.

Let $\sigma=\lambda \max _{w}\left|f^{\prime}(w)\right|$. For the class of fully-discrete $\alpha$ schemes when $m=2$, equipped with above lemmas, we have obtained the following entropy convergence result. The proof is similar to the one given by Jiang [7] for van Leer's flux limiter schemes and we omit the proof.

THEOREM 3.8. The numerical solutions of the schemes (2.9)-(2.10), for the convex problems (1.1), converge to the entropy solution provided that $g^{E}(\cdot, \cdot)$ is a numerical flux given by (2.23), and $\sigma$ is sufficiently small.

Finally, we finish this section by presenting the proof of Lemma 3.6.

Proof of Lemma 3.6. In the following, we keep the same notations $f_{j+\frac{1}{2}}^{ \pm}$and $r_{j}^{ \pm}$ for $\left\{v_{j}\right\}$ instead of $\left\{u_{j}\right\}$. We also use

$$
f_{j+\frac{1}{2}}^{\prime}:=\frac{f\left(v_{j+1}\right)-f\left(v_{j}\right)}{v_{j+1}-v_{j}}
$$

to denote the divided difference.

To justify the inequality (3.14), it suffices to show the following inequality:

$$
\int_{L}^{R} g_{\Gamma}(w) d w-\sum_{j=I}^{J-1} \int_{v_{j}}^{v_{j+1}} f\left[w ; v_{j}, v_{j+1}\right] d w \leq \sum_{j=I+1}^{J-1} \alpha_{j} S_{j-1, j, j+1} .
$$

Without loss of generality, Let $v_{s}$ be a sonic point $\left(f^{\prime}\left(v_{s}\right)=0\right)$ such that $v_{k} \leq$ $v_{s} \leq v_{k+1}$ for some integer $k$ with $I \leq k \leq J-1$. Then for any $g^{E}(\cdot, \cdot)$ given by (2.23), we have

$$
f_{j+\frac{1}{2}}^{+}=0, \quad \text { for } I \leq j \leq k-1
$$




$$
\begin{gathered}
f_{j+\frac{1}{2}}^{+}=f_{j+\frac{1}{2}}^{\prime} v_{j+\frac{1}{2}}, \quad \text { for } J-1 \geq j \geq k+1 ; \\
f_{j+\frac{1}{2}}^{-}=0, \quad \text { for } J-1 \geq j \geq k+1
\end{gathered}
$$

and

$$
f_{j+\frac{1}{2}}^{-}=f_{j+\frac{1}{2}}^{\prime} v_{j+\frac{1}{2}}, \quad \text { for } I \leq j \leq k-1
$$

To enhance the readability, we further simplify the notations. Denote $f_{j}:=f\left(v_{j}\right)$, $f_{s}:=f\left(v_{s}\right), v_{j \pm \frac{1}{2}}:=\Delta v_{j \pm \frac{1}{2}}= \pm\left(v_{j \pm 1}-v_{j}\right), v_{s+\frac{1}{2}}:=v_{k+1}-v_{s}, v_{s-\frac{1}{2}}:=v_{s}-v_{k}$, $\bar{v}_{j \pm \frac{1}{2}}:=\Delta \bar{v}_{j \pm \frac{1}{2}}, \tilde{v}_{j \pm \frac{1}{2}}:=\Delta \tilde{v}_{j \pm \frac{1}{2}}, f_{s+\frac{1}{2}}^{\prime}:=\left(f_{k+1}-f_{s}\right) / v_{s+\frac{1}{2}}$, and $f_{s-\frac{1}{2}}^{\prime}:=\left(f_{s}-\right.$ $\left.f_{k}\right) / v_{s-\frac{1}{2}}$. Then, by (3.1), we have

$$
\begin{aligned}
\operatorname{LHS} \text { of }(3.16) & =\sum_{j=I}^{J-1} g_{j+\frac{1}{2}} \bar{v}_{j+\frac{1}{2}}-\sum_{j=I}^{J-1} \int_{v_{j}}^{v_{j+1}} f\left[w ; v_{j}, v_{j+1}\right] d w \\
& =\sum_{j=I}^{J-1} g_{j+\frac{1}{2}} \frac{v_{j+\frac{1}{2}}+\tilde{v}_{j+\frac{1}{2}}}{2}-\sum_{j=I}^{J-1} \frac{f_{j}+f_{j+1}}{2} v_{j+\frac{1}{2}} \\
& =\frac{1}{2}\left(P_{(j \leq k-2)}+P_{k-1}+P_{k}+P_{k+1}+P_{(j \geq k+2)}\right),
\end{aligned}
$$

and the definitions of $P_{(j \leq k-2)}, P_{k-1}, P_{k}, P_{k+1}$ and $P_{(j \geq k+2)}$ will be given shortly.

Recall the numerical flux is defined by

$$
\begin{aligned}
g_{j+\frac{1}{2}}= & g_{j+\frac{1}{2}}^{E}-\alpha\left(f_{j+\frac{3}{2}}^{-}\right)^{(1)}-\left(\frac{1}{2}-\alpha\right)\left(f_{j+\frac{1}{2}}^{-}\right)^{(0)} \\
& \left(\frac{1}{2}-\alpha\right)\left(f_{j+\frac{1}{2}}^{+}\right)^{(0)}+\alpha\left(f_{j-\frac{1}{2}}^{+}\right)^{(-1)},
\end{aligned}
$$

and using the increment form (2.15)-(2.17), we have

$$
\tilde{v}_{j+\frac{1}{2}}=v_{j+\frac{1}{2}}-\left(C_{j+\frac{1}{2}}+D_{j+\frac{1}{2}}\right) v_{j+\frac{1}{2}}+D_{j+\frac{3}{2}} v_{j+\frac{3}{2}}+C_{j-\frac{1}{2}} v_{j-\frac{1}{2}} \geq 0
$$

Let

$$
\begin{gathered}
d_{j}:=-\left(\frac{1}{2}+2 \alpha\right) f_{j+\frac{1}{2}}^{\prime} v_{j+\frac{1}{2}}^{2}-\left(\frac{1}{2}-\alpha\right) f_{j-\frac{1}{2}}^{\prime} v_{j-\frac{1}{2}} v_{j+\frac{1}{2}}, \\
T:=\left[f_{k-1}-\alpha f_{k-\frac{1}{2}}^{\prime} v_{k-\frac{1}{2}}-\left(\frac{1}{2}-\alpha\right) f_{k-\frac{3}{2}}^{\prime} v_{k-\frac{3}{2}}\right] D_{k-\frac{1}{2}} v_{k-\frac{1}{2}},
\end{gathered}
$$

and

$$
T_{(k \leq k-2)}:=-\alpha f_{k-\frac{1}{2}}^{\prime} v_{k-\frac{1}{2}}^{2}+T \text {. }
$$

With these definitions and the convexity of the flux in mind, we have derived the following estimates. 
(3.18)

$$
\begin{aligned}
& P_{(j \leq k-2)}:=\sum_{j=I}^{k-2}\left\{g_{j+\frac{1}{2}}\left[v_{j+\frac{1}{2}}+\tilde{v}_{j+\frac{1}{2}}\right]-\left(f_{j}+f_{j+1}\right) v_{j+\frac{1}{2}}\right\} \\
& =\sum_{j=I}^{k-2}\left\{[ g _ { j + \frac { 1 } { 2 } } ^ { E } - \alpha ( f _ { j + \frac { 3 } { 2 } } ^ { - } ) ^ { ( 1 ) } - ( \frac { 1 } { 2 } - \alpha ) ( f _ { j + \frac { 1 } { 2 } } ^ { - } ) ^ { ( 0 ) } ] \left[2 v_{j+\frac{1}{2}}-D_{j+\frac{1}{2}} v_{j+\frac{1}{2}}\right.\right. \\
& \left.\left.+D_{j+\frac{3}{2}} v_{j+\frac{3}{2}}\right]-\left(f_{j}+f_{j+1}\right) v_{j+\frac{1}{2}}\right\} \\
& \leq \sum_{j=I}^{k-2}\left\{[ g _ { j + \frac { 1 } { 2 } } ^ { E } - \alpha f _ { j + \frac { 3 } { 2 } } ^ { - } - ( \frac { 1 } { 2 } - \alpha ) f _ { j + \frac { 1 } { 2 } } ^ { - } ] \left[2 v_{j+\frac{1}{2}}-D_{j+\frac{1}{2}} v_{j+\frac{1}{2}}\right.\right. \\
& \left.\left.+D_{j+\frac{3}{2}} v_{j+\frac{3}{2}}\right]-\left(f_{j}+f_{j+1}\right) v_{j+\frac{1}{2}}\right\} \\
& =\sum_{j=I}^{k-2}\left\{\left[2 f_{j+1}-\left(f_{j}+f_{j+1}\right)\right] v_{j+\frac{1}{2}}-2 \alpha f_{j+\frac{3}{2}}^{\prime} v_{j+\frac{3}{2}} v_{j+\frac{1}{2}}\right. \\
& \left.-(1-2 \alpha) f_{j+\frac{1}{2}}^{\prime} v_{j+\frac{1}{2}}^{2}\right\} \\
& -\sum_{j=I}^{k-2}\left[f_{j+1} v_{j+\frac{1}{2}}-\alpha f_{j+\frac{3}{2}}^{\prime} v_{j+\frac{3}{2}} v_{j+\frac{1}{2}}-\left(\frac{1}{2}-\alpha\right) f_{j+\frac{1}{2}}^{\prime} v_{j+\frac{1}{2}}^{2}\right] D_{j+\frac{1}{2}} \\
& +\sum_{j=I}^{k-2}\left[f_{j+1} v_{j+\frac{3}{2}}-\alpha f_{j+\frac{3}{2}}^{\prime} v_{j+\frac{3}{2}}^{2}-\left(\frac{1}{2}-\alpha\right) f_{j+\frac{1}{2}}^{\prime} v_{j+\frac{1}{2}} v_{j+\frac{3}{2}}\right] D_{j+\frac{3}{2}} \\
& =\sum_{j=I}^{k-2}\left[-2 \alpha f_{j+\frac{3}{2}}^{\prime} v_{j+\frac{3}{2}} v_{j+\frac{1}{2}}+2 \alpha f_{j+\frac{1}{2}}^{\prime} v_{j+\frac{1}{2}}^{2}\right]+\sum_{j=I}^{k-2}\left[-f_{j+1} v_{j+\frac{1}{2}}\right. \\
& +\alpha f_{j+\frac{3}{2}}^{\prime} v_{j+\frac{3}{2}} v_{j+\frac{1}{2}}+\left(\frac{1}{2}-\alpha\right) f_{j+\frac{1}{2}}^{\prime} v_{j+\frac{1}{2}}^{2}+f_{j} v_{j+\frac{1}{2}} \\
& \left.-\alpha f_{j+\frac{1}{2}}^{\prime} v_{j+\frac{1}{2}}^{2}-\left(\frac{1}{2}-\alpha\right) f_{j-\frac{1}{2}}^{\prime} v_{j-\frac{1}{2}} v_{j+\frac{1}{2}}\right] D_{j+\frac{1}{2}}+T \\
& \leq \sum_{j=I}^{k-2} \alpha f_{j+\frac{3}{2}}^{\prime}\left(v_{j+\frac{3}{2}}-v_{j+\frac{1}{2}}\right)^{2}-\alpha f_{k-\frac{1}{2}}^{\prime} v_{k-\frac{1}{2}}^{2}+\sum_{j=I}^{k-2}\left[-\left(\frac{1}{2}+2 \alpha\right) f_{j+\frac{1}{2}}^{\prime} v_{j+\frac{1}{2}}^{2}\right. \\
& \left.+\alpha f_{j+\frac{3}{2}}^{\prime} v_{j+\frac{3}{2}} v_{j+\frac{1}{2}}-\left(\frac{1}{2}-\alpha\right) f_{j-\frac{1}{2}}^{\prime} v_{j-\frac{1}{2}} v_{j+\frac{1}{2}}\right] D_{j+\frac{1}{2}}+T \\
& \leq \sum_{j=I}^{k-2}\left[-\left(\frac{1}{2}+2 \alpha\right) f_{j+\frac{1}{2}}^{\prime} v_{j+\frac{1}{2}}^{2}+\alpha f_{j+\frac{3}{2}}^{\prime} v_{j+\frac{3}{2}} v_{j+\frac{1}{2}}\right. \\
& \left.-\left(\frac{1}{2}-\alpha\right) f_{j-\frac{1}{2}}^{\prime} v_{j-\frac{1}{2}} v_{j+\frac{1}{2}}\right] D_{j+\frac{1}{2}}+T_{(j \leq k-2)} \\
& \leq \sum_{j=I}^{k-2} d_{j} D_{j+\frac{1}{2}}+T_{(j \leq k-2)} \text {. }
\end{aligned}
$$


Similarly, notice that by writing

$$
\begin{aligned}
& -2 \alpha \sum_{j=k+2}^{J-1} f_{j+\frac{1}{2}}^{\prime} v_{j+\frac{1}{2}}^{2} \\
& =-\alpha \sum_{j=k+2}^{J-1} f_{j+\frac{1}{2}}^{\prime} v_{j+\frac{1}{2}}^{2}-\alpha \sum_{j=k+2}^{J-1} f_{j-\frac{1}{2}}^{\prime} v_{j-\frac{1}{2}}^{2}+\alpha f_{k+\frac{3}{2}}^{\prime} v_{k+\frac{3}{2}}^{2},
\end{aligned}
$$

we obtain

$$
\begin{aligned}
& P_{(j \geq k+2)}:=\sum_{j=k+2}^{J-1}\left\{g_{j+\frac{1}{2}}\left[v_{j+\frac{1}{2}}+\tilde{v}_{j+\frac{1}{2}}\right]-\left(f_{j}+f_{j+1}\right) v_{j+\frac{1}{2}}\right\} \\
= & \sum_{j=k+2}^{J-1}\left\{[ g _ { j + \frac { 1 } { 2 } } ^ { E } + ( \frac { 1 } { 2 } - \alpha ) ( f _ { j + \frac { 1 } { 2 } } ^ { + } ) ^ { ( 0 ) } + \alpha ( f _ { j - \frac { 1 } { 2 } } ^ { + } ) ^ { ( - 1 ) } ] \left[2 v_{j+\frac{1}{2}}\right.\right. \\
& \left.\left.-C_{j+\frac{1}{2}} v_{j+\frac{1}{2}}+C_{j-\frac{1}{2}} v_{j-\frac{1}{2}}\right]-\left(f_{j}+f_{j+1}\right) v_{j+\frac{1}{2}}\right\} \\
\leq & \sum_{j=k+2}^{J-1}\left\{[ f _ { j } + ( \frac { 1 } { 2 } - \alpha ) f _ { j + \frac { 1 } { 2 } } ^ { + } + \alpha f _ { j - \frac { 1 } { 2 } } ^ { + } ] \left[2 v_{j+\frac{1}{2}}\right.\right. \\
& \left.\left.-C_{j+\frac{1}{2}} v_{j+\frac{1}{2}}+C_{j-\frac{1}{2}} v_{j-\frac{1}{2}}\right]-\left(f_{j}+f_{j+1}\right) v_{j+\frac{1}{2}}\right\} \\
\leq & \sum_{j=k+2}^{J-1}\left[\left(\frac{1}{2}+2 \alpha\right) f_{j+\frac{1}{2}}^{\prime} v_{j+\frac{1}{2}}^{2}+\left(\frac{1}{2}-\alpha\right) f_{j+\frac{3}{2}}^{\prime} v_{j+\frac{1}{2}} v_{j+\frac{3}{2}}\right] C_{j+\frac{1}{2}}+T_{(j \geq k+2)} \\
= & \sum_{j=k+2}^{J-1} c_{j} C_{j+\frac{1}{2}}+T_{(j \geq k+2)},
\end{aligned}
$$

where

$$
c_{j}:=\left(\frac{1}{2}+2 \alpha\right) f_{j+\frac{1}{2}}^{\prime} v_{j+\frac{1}{2}}^{2}+\left(\frac{1}{2}-\alpha\right) f_{j+\frac{3}{2}}^{\prime} v_{j+\frac{1}{2}} v_{j+\frac{3}{2}}
$$

and

$$
\begin{aligned}
T_{(j \geq k+2)}:= & \alpha f_{k+\frac{3}{2}}^{\prime} v_{k+\frac{3}{2}}^{2}+\left[f_{k+2} v_{k+\frac{3}{2}}+\left(\frac{1}{2}-\alpha\right) f_{k+\frac{5}{2}}^{\prime} v_{k+\frac{5}{2}} v_{k+\frac{3}{2}}\right. \\
& \left.+\alpha f_{k+\frac{3}{2}}^{\prime} v_{k+\frac{3}{2}}^{2}\right] C_{k+\frac{3}{2}} .
\end{aligned}
$$

Now, we compute the $(k-1)$ th, $k$ th and $(k+1)$ th terms of the LHS of $(3.16)$ 
defined by $P_{k-1}, P_{k}$ and $P_{k+1}$ respectively as follows.

$$
\begin{aligned}
P_{k-1}:= & \left.g_{k-\frac{1}{2}}^{E}+\alpha\left(f_{k+\frac{1}{2}}^{-}\right)^{(1)}-\left(\frac{1}{2}-\alpha\right)\left(f_{k-\frac{1}{2}}^{-}\right)^{(0)}\right]\left[2 v_{k-\frac{1}{2}}-D_{k-\frac{1}{2}} v_{k-\frac{1}{2}}\right. \\
& +D_{k+\frac{1}{2}} v_{k+\frac{1}{2}}-\left(f_{k-1}+f_{k}\right) v_{k-\frac{1}{2}} \\
\leq & {\left[f_{k}-\alpha f_{k+\frac{1}{2}}^{-}-\left(\frac{1}{2}-\alpha\right) f_{k-\frac{1}{2}}^{-}\right]\left[2 v_{k-\frac{1}{2}}-D_{k-\frac{1}{2}} v_{k-\frac{1}{2}}\right.} \\
& \left.+D_{k+\frac{1}{2}} v_{k+\frac{1}{2}}\right]-\left(f_{k-1}+f_{k}\right) v_{k-\frac{1}{2}} \\
= & -2 \alpha f_{s-\frac{1}{2}}^{\prime} v_{s-\frac{1}{2}} v_{k-\frac{1}{2}}+2 \alpha f_{k-\frac{1}{2}}^{\prime} v_{k-\frac{1}{2}}^{2}+\left[-f_{k} v_{k-\frac{1}{2}}+\alpha f_{s-\frac{1}{2}}^{\prime} v_{s-\frac{1}{2}} v_{k-\frac{1}{2}}\right. \\
& \left.+\left(\frac{1}{2}-\alpha\right) f_{k-\frac{1}{2}}^{\prime} v_{k-\frac{1}{2}}^{2}\right] D_{k-\frac{1}{2}}+\left[f_{k} v_{k+\frac{1}{2}}-\alpha f_{s-\frac{1}{2}}^{\prime} v_{s-\frac{1}{2}} v_{k+\frac{1}{2}}\right. \\
& \left.-\left(\frac{1}{2}-\alpha\right) f_{k-\frac{1}{2}}^{\prime} v_{k-\frac{1}{2}} v_{k+\frac{1}{2}}\right] D_{k+\frac{1}{2}}, \\
P_{k}:= & g_{k+\frac{1}{2}}^{E}\left[2 v_{k+\frac{1}{2}}-D_{k+\frac{1}{2}} v_{k+\frac{1}{2}}-C_{k+\frac{1}{2}} v_{k+\frac{1}{2}}\right]-\left(f_{k}+f_{k+1}\right) v_{k+\frac{1}{2}} \\
= & f_{s}\left[2 v_{k+\frac{1}{2}}-D_{k+\frac{1}{2}} v_{k+\frac{1}{2}}-C_{k+\frac{1}{2}} v_{k+\frac{1}{2}}\right]-\left(f_{k}+f_{k+1}\right) v_{k+\frac{1}{2}} \\
= & f_{s-\frac{1}{2}}^{\prime} v_{s-\frac{1}{2}} v_{k+\frac{1}{2}}-f_{s+\frac{1}{2}}^{\prime} v_{s+\frac{1}{2}} v_{k+\frac{1}{2}}-f_{s} v_{k+\frac{1}{2}} D_{k+\frac{1}{2}}-f_{s} v_{k+\frac{1}{2}} C_{k+\frac{1}{2}},
\end{aligned}
$$

and

$$
\begin{aligned}
P_{k+1}:= & \left.g_{k+\frac{3}{2}}^{E}+\left(\frac{1}{2}-\alpha\right)\left(f_{k+\frac{3}{2}}^{+}\right)^{(0)}+\alpha\left(f_{k+\frac{1}{2}}^{+}\right)^{(-1)}\right]\left[2 v_{k+\frac{3}{2}}-C_{k+\frac{3}{2}} v_{k+\frac{3}{2}}\right. \\
& \left.+C_{k+\frac{1}{2}} v_{k+\frac{1}{2}}\right]-\left(f_{k+1}+f_{k+2}\right) v_{k+\frac{3}{2}} \\
\leq & {\left[f_{k+1}+\left(\frac{1}{2}-\alpha\right) f_{k+\frac{3}{2}}^{+}+\alpha f_{k+\frac{1}{2}}^{+}\right]\left[2 v_{k+\frac{3}{2}}-C_{k+\frac{3}{2}} v_{k+\frac{3}{2}}\right.} \\
& \left.+C_{k+\frac{1}{2}} v_{k+\frac{1}{2}}\right]-\left(f_{k+1}+f_{k+2}\right) v_{k+\frac{3}{2}} \\
= & -2 \alpha f_{k+\frac{3}{2}}^{\prime} v_{k+\frac{3}{2}}^{2}+2 \alpha f_{s+\frac{1}{2}}^{\prime} v_{s+\frac{1}{2}} v_{k+\frac{3}{2}}+\left[-f_{k+1} v_{k+\frac{3}{2}}\right. \\
& \left.-\left(\frac{1}{2}-\alpha\right) f_{k+\frac{3}{2}}^{\prime} v_{k+\frac{3}{2}}^{2}-\alpha f_{s+\frac{1}{2}}^{\prime} v_{s+\frac{1}{2}} v_{k+\frac{3}{2}}\right] C_{k+\frac{3}{2}}+\left[f_{k+1} v_{k+\frac{1}{2}}\right. \\
& \left.+\left(\frac{1}{2}-\alpha\right) f_{k+\frac{3}{2}}^{\prime} v_{k+\frac{1}{2}} v_{k+\frac{3}{2}}+\alpha f_{s+\frac{1}{2}}^{\prime} v_{s+\frac{1}{2}} v_{k+\frac{1}{2}}\right] C_{k+\frac{1}{2}}
\end{aligned}
$$

Next, we combine the estimates of $T_{(j \leq k-2)}, T_{(j \geq k+2)}, P_{k-1}, P_{k}$, and $P_{k+1}$ into one estimate. But first, we let

$$
\begin{gathered}
d_{k-1}=-\left(\frac{1}{2}+2 \alpha\right) f_{k-\frac{1}{2}}^{\prime} v_{k-\frac{1}{2}}^{2}-\left(\frac{1}{2}-\alpha\right) f_{k-\frac{3}{2}}^{\prime} v_{k-\frac{3}{2}} v_{k-\frac{1}{2}}+\alpha f_{s-\frac{1}{2}}^{\prime} v_{s-\frac{1}{2}} v_{k-\frac{1}{2}}, \\
d_{k}=-\left(\frac{1}{2}-\alpha\right) f_{k-\frac{1}{2}}^{\prime} v_{k-\frac{1}{2}} v_{k+\frac{1}{2}}-(1+\alpha) f_{s-\frac{1}{2}}^{\prime} v_{s-\frac{1}{2}} v_{k+\frac{1}{2}} \\
c_{k}=(1+\alpha) f_{s+\frac{1}{2}}^{\prime} v_{s+\frac{1}{2}} v_{k+\frac{1}{2}}+\left(\frac{1}{2}-\alpha\right) f_{k+\frac{3}{2}}^{\prime} v_{k+\frac{1}{2}} v_{k+\frac{3}{2}}
\end{gathered}
$$

and

$$
c_{k+1}=\left(\frac{1}{2}+2 \alpha\right) f_{k+\frac{3}{2}}^{\prime} v_{k+\frac{3}{2}}^{2}+\left(\frac{1}{2}-\alpha\right) f_{k+\frac{5}{2}}^{\prime} v_{k+\frac{3}{2}} v_{k+\frac{5}{2}}-\alpha f_{s+\frac{1}{2}}^{\prime} v_{s+\frac{1}{2}} v_{k+\frac{3}{2}} .
$$


Using the facts that $v_{k+\frac{1}{2}}=v_{s+\frac{1}{2}}+v_{s-\frac{1}{2}}, f_{s-\frac{1}{2}}^{\prime} v_{s-\frac{1}{2}} v_{k+\frac{1}{2}}-f_{s-\frac{1}{2}}^{\prime} v_{s-\frac{1}{2}}^{2}=$ $f_{s-\frac{1}{2}}^{\prime} v_{s-\frac{1}{2}} v_{s+\frac{1}{2}}$ and $-f_{s+\frac{1}{2}}^{\prime} v_{s+\frac{1}{2}} v_{k+\frac{1}{2}}+f_{s+\frac{1}{2}}^{\prime} v_{s+\frac{1}{2}}^{2}=-f_{s+\frac{1}{2}}^{\prime} v_{s-\frac{1}{2}} v_{s+\frac{1}{2}}$, we have

$$
\begin{aligned}
& T(k):=T_{(j \leq k-2)}+T_{(j \geq k+2)}+P_{k-1}+P_{k}+P_{k+1} \\
\leq & -\alpha f_{k-\frac{1}{2}}^{\prime} v_{k-\frac{1}{2}}^{2}+\left[f_{k-1}-\alpha f_{k-\frac{1}{2}}^{\prime} v_{k-\frac{1}{2}}-\left(\frac{1}{2}-\alpha\right) f_{k-\frac{3}{2}}^{\prime} v_{k-\frac{3}{2}}\right] D_{k-\frac{1}{2}} v_{k-\frac{1}{2}} \\
& +\alpha f_{k+\frac{3}{2}}^{\prime} v_{k+\frac{3}{2}}^{2}+\left[f_{k+2} v_{k+\frac{3}{2}}+\left(\frac{1}{2}-\alpha\right) f_{k+\frac{5}{2}}^{\prime} v_{k+\frac{3}{2}} v_{k+\frac{5}{2}}+\alpha f_{k+\frac{3}{2}}^{\prime} v_{k+\frac{3}{2}}^{2}\right] C_{k+\frac{3}{2}} \\
& -2 \alpha f_{s-\frac{1}{2}}^{\prime} v_{s-\frac{1}{2}} v_{k-\frac{1}{2}}+2 \alpha f_{k-\frac{1}{2}}^{\prime} v_{k-\frac{1}{2}}^{2}+\left[-f_{k} v_{k-\frac{1}{2}}+\alpha f_{s-\frac{1}{2}}^{\prime} v_{s-\frac{1}{2}} v_{k-\frac{1}{2}}\right. \\
& \left.+\left(\frac{1}{2}-\alpha\right) f_{k-\frac{1}{2}}^{\prime} v_{k-\frac{1}{2}}^{2}\right] D_{k-\frac{1}{2}}+\left[f_{k} v_{k+\frac{1}{2}}-\alpha f_{s-\frac{1}{2}}^{\prime} v_{s-\frac{1}{2}} v_{k+\frac{1}{2}}\right. \\
& \left.-\left(\frac{1}{2}-\alpha\right) f_{k-\frac{1}{2}}^{\prime} v_{k-\frac{1}{2}} v_{k+\frac{1}{2}}\right] D_{k+\frac{1}{2}} \\
& +f_{s-\frac{1}{2}}^{\prime} v_{s-\frac{1}{2}} v_{k+\frac{1}{2}}-f_{s+\frac{1}{2}}^{\prime} v_{s+\frac{1}{2}} v_{k+\frac{1}{2}}-f_{s} v_{k+\frac{1}{2}} D_{k+\frac{1}{2}}-f_{s} v_{k+\frac{1}{2}} C_{k+\frac{1}{2}} \\
& -2 \alpha f_{k+\frac{3}{2}}^{\prime} v_{k+\frac{3}{2}}^{2}+2 \alpha f_{s+\frac{1}{2}}^{\prime} v_{s+\frac{1}{2}} v_{k+\frac{3}{2}} \\
& +\left[-f_{k+1} v_{k+\frac{3}{2}}-\left(\frac{1}{2}-\alpha\right) f_{k+\frac{3}{2}}^{\prime} v_{k+\frac{3}{2}}^{2}-\alpha f_{s+\frac{1}{2}}^{\prime} v_{s+\frac{1}{2}} v_{k+\frac{3}{2}}\right] C_{k+\frac{3}{2}} \\
& +\left[f_{k+1} v_{k+\frac{1}{2}}+\left(\frac{1}{2}-\alpha\right) f_{k+\frac{3}{2}}^{\prime} v_{k+\frac{1}{2}} v_{k+\frac{3}{2}}+\alpha f_{s+\frac{1}{2}}^{\prime} v_{s+\frac{1}{2}} v_{k+\frac{1}{2}}\right] C_{k+\frac{1}{2}} \\
= & T_{1}(k)+T_{2}(k) \\
\leq & T_{2}(k),
\end{aligned}
$$

where

$$
\begin{aligned}
T_{1}(k):= & \alpha f_{k-\frac{1}{2}}^{\prime} v_{k-\frac{1}{2}}^{2}-2 \alpha f_{s-\frac{1}{2}}^{\prime} v_{s-\frac{1}{2}} v_{k-\frac{1}{2}}+f_{s-\frac{1}{2}}^{\prime} v_{s-\frac{1}{2}} v_{k+\frac{1}{2}} \\
& -f_{s+\frac{1}{2}}^{\prime} v_{s+\frac{1}{2}} v_{k+\frac{1}{2}}-\alpha f_{k+\frac{3}{2}}^{\prime} v_{k+\frac{3}{2}}^{2}+2 \alpha f_{s+\frac{1}{2}}^{\prime} v_{s+\frac{1}{2}} v_{k+\frac{3}{2}} \\
= & \alpha f_{k-\frac{1}{2}}^{\prime} v_{k-\frac{1}{2}}^{2}+\alpha f_{s-\frac{1}{2}}^{\prime}\left(v_{s-\frac{1}{2}}-v_{k-\frac{1}{2}}\right)^{2}-\alpha f_{s-\frac{1}{2}}^{\prime} v_{s-\frac{1}{2}}^{2}-\alpha f_{s-\frac{1}{2}}^{\prime} v_{k-\frac{1}{2}}^{2} \\
& +f_{s-\frac{1}{2}}^{\prime} v_{s-\frac{1}{2}} v_{k+\frac{1}{2}}-f_{s+\frac{1}{2}}^{\prime} v_{s+\frac{1}{2}} v_{k+\frac{1}{2}}-\alpha f_{k+\frac{3}{2}}^{\prime} v_{k+\frac{3}{2}}^{2}+2 \alpha f_{s+\frac{1}{2}}^{\prime} v_{s+\frac{1}{2}} v_{k+\frac{3}{2}} \\
\leq & \alpha\left(f_{k-\frac{1}{2}}^{\prime}-f_{s-\frac{1}{2}}^{\prime}\right) v_{k-\frac{1}{2}}^{2}+(1-\alpha) f_{s-\frac{1}{2}}^{\prime} v_{s-\frac{1}{2}}^{2}+f_{s-\frac{1}{2}}^{\prime} v_{s-\frac{1}{2}} v_{s+\frac{1}{2}} \\
& -\alpha f_{s+\frac{1}{2}}^{\prime}\left(v_{s+\frac{1}{2}}-v_{k+\frac{3}{2}}\right)^{2}-(1-\alpha) f_{s+\frac{1}{2}}^{\prime} v_{s+\frac{1}{2}}^{2}-f_{s+\frac{1}{2}}^{\prime} v_{s-\frac{1}{2}} v_{s+\frac{1}{2}} \\
\leq & 0
\end{aligned}
$$

and

$$
T_{2}(k):=d_{k-1} D_{k-\frac{1}{2}}+d_{k} D_{k+\frac{1}{2}}+c_{k} C_{k+\frac{1}{2}}+c_{k+1} C_{k+\frac{3}{2}}
$$


Finally, using (3.17), (3.18), (3.19) and (3.20), we have

$$
\begin{aligned}
\text { LHS of }(3.16) & =\sum_{j=I}^{J-1} g_{j+\frac{1}{2}} \bar{v}_{j+\frac{1}{2}}-\sum_{j=I}^{J-1} \int_{v_{j}}^{v_{j+1}} f\left[w ; v_{j}, v_{j+1}\right] d w \\
& =\frac{1}{2}\left(P_{(j \leq k-2)}+P_{k-1}+P_{k}+P_{k+1}+P_{(j \geq k+2)}\right) \\
& \leq \frac{1}{2}\left\{\sum_{j=I}^{k-2} d_{j} D_{j+\frac{1}{2}}+\sum_{j=k+2}^{J-1} c_{j} C_{j+\frac{1}{2}}+T_{2}(k)\right\} \\
& =\frac{1}{2}\left\{\sum_{j=I}^{k} d_{j} D_{j+\frac{1}{2}}+\sum_{j=k}^{J-1} c_{j} C_{j+\frac{1}{2}}\right\} .
\end{aligned}
$$

Clearly, for sufficiently small $\sigma_{\Gamma}$, it is feasible that

$$
\text { LHS of }(3.16) \leq \frac{1}{2} \sum_{j=I+1}^{J-1} S_{j-1, j, j+1} \leq \sum_{j=I+1}^{J-1} \alpha_{j} S_{j-1, j, j+1} .
$$

Thus, we have completed the proof of Lemma 3.6.

\section{REFERENCES}

[1] S. Chakravarthy and S. Osher, A New Class of High Accuracy TVD Schemes for Hyperbolic Conservation Laws, AIAA paper, 1-11, 23rd Aerospace Science Meeting (1985), Reno, Nevada.

[2] S. Chakravarthy and S. Osher, Computing with High Resolution Upwind Schemes for Hyperbolic Equations, Proceedings of AMS-SIAM 1983.

[3] B. EngQuist AND S. Osher, Stable and entropy satisfying approximations for transonic flow calculations, Math. Comp., 34 (1980), pp. 45-75.

[4] S. K. Godunov, Finite-difference method for numerical computation of discontinuous solutions of the equations of fluid dynamics, Mat. Sbornik, 47 (1959), pp. 271-306.

[5] A. Harten, High resolution schemes for hyperbolic conservative laws, J. Comput. Phys., 49 (1983), pp. 357-393.

[6] N. JiAng, The Convergence of $\alpha$ Schemes for Conservation Laws I: Semi-Discrete Case, Methods and Applications of Analysis, 19:4 (2012), pp. 341-358.

[7] N. JiAng, On the Convergence of Fully-discrete High-Resolution Schemes with van Leer's flux limiter for Conservation laws, Methods and Applications of Analysis, 16:3 (2009), pp. 403422 .

[8] N. JIANG, On the convergence of Semi-discrete High Resolution Schemes with Superbee flux limiter for Conservation laws, Series in Contemporary Applied Mathematics CAM 18, Hyperbolic Problems (Theory, Numerics and Applications) ISBN 978-7-04-034536-0, Vol. 2 (2012), pp. 431-438.

[9] N. Jiang And H. YAng, On Convergence of Semi-Discrete High Resolution Schemes with van Leer's Flux Limiter for Conservation Laws, Methods and Applications of Analysis, 12:1 (2005), pp. 89-102.

[10] P. Lax And B. Wendroff, Systems of conservation laws, Comm. Pure Appl. Math., 13 (1960), pp. $217-237$.

[11] P. LAx, Hyperbolic Systems of Conservation Laws and the Mathematical Theory of Shock Waves, SIAM Regional Conference Series in Applied Mathematics, 11 (1972).

[12] S. Osher And S. Chakravarthy, Very High Order Accurate TVD Schemes, Journal of Oscillation theory, computation, and methods of compensated compactness, (1986), pp. 229-274.

[13] S. Osher AND S. Chakravarthy, High resolution schemes and entropy condition, SIAM J. Numer. Anal., 21 (1984), pp. 955-984.

[14] P. K. SweBy, High resolution schemes using flux limiters for hyperbolic conservation laws, SIAM J. Numer. Anal., 21 (1984), pp. 995-1011.

[15] E. TADMOR, Convenient total variation diminishing conditions for nonlinear difference schemes, SIAM J. Numer. Anal., 25 (1988), pp. 1002-1014. 
[16] H. YANG, On Wavewise Entropy Inequalities for High-Resolution Schemes I: The SemiDiscrete Case, Math. Comp., 65 (1996), pp. 45-67.

[17] H. Yang, On Wavewise Entropy Inequalities for High Resolution Schemes II: Fully Discrete MUSCL Schemes with Exact Evolution in Small Time, SIAM. J. Numer. Anal., 36:1 (1999), pp. 1-31.

[18] H. YAng AND N. JiAng, On Wavewise Entropy Inequalities for High-Resolution Schemes with Source Terms I: The Semi-Discrete Case, Methods and Applications of Analysis, 10:4 (2003), pp. 487-512. 
